\title{
Health care operations management
}

\author{
Michael Carter • Erwin W. Hans • Rainer Kolisch
}

Published online: 14 February 2012

C The Author(s) 2012. This article is published with open access at Springerlink.com

Health care operations management has become a major topic for health care service providers and society. Operations research already has and further will make considerable contributions for the effective and efficient delivery of health care services. This special issue collects seven carefully selected papers dealing with optimization and decision analysis problems in the field of health care operations management. The papers cover a considerable range of health care problems including location planning for hospital and health services (Mestre et al., Zhang et al.), organization of hospital resources (Vanberkel et al., Hulshof et al.), surgery scheduling (Marques et al., Herring and Herrmann) and treatment scheduling (Schimmelpfeng et al.). These problems are addressed within a number of different health care environments such as hospitals, preventive care, outpatient clinics and rehabilitation hospitals. The operations research techniques which are employed are mixed-integer linear programming, stochastic dynamic programming, hierarchical decomposition, queueing theory, simulation and choice models. The special issue thus covers a broad range of problems, environments and techniques. It is noteworthy that all papers are either treating a real life problem or are inspired by the latter, which demonstrates the problem-driven

\footnotetext{
M. Carter

Department of Mechanical and Industrial Engineering, University of Toronto, Toronto, Canada e-mail: carter@mie.utoronto.ca

E. W. Hans

CHOIR Center for Healthcare Optimization and Research,

School of Management and Governance, University of Twente, Enschede, The Netherlands e-mail: e.w.hans@utwente.nl

R. Kolisch ( $)$

TUM School of Management, Technische Universität München, München, Germany

e-mail: rainer.kolisch@wi.tum.de
} 
nature of health care operations research. In particular, this special issue addresses the following topics.

\section{Location, supply and referrals in planned hospital systems}

The paper of A.M. Mestre, M.D. Oliveira and A. Barbosa-Póvoa revolves around making decisions on geographic location and organization of hospital services to achieve equitable health care access and efficient health care delivery. A hierarchical multiservice mathematical programming model is proposed, which simultaneously considers the multiservice structure of hospitals' production, the hospital network configuration costs, the articulation between different hospital services and between hospital units, and the ascendant and descendent flows related to two-way referrals of patients in the hospital hierarchy. The model is applied to the real case of the South region of the Portuguese National Health Service.

\section{Preventive health care facility network design}

Y. Zhang, O. Berman and V. Verter contribute an interesting study of the design of preventive healthcare facility networks, where the aim is to maximize participation of the clientele. Clientele has access to preventive health care in their region on a voluntary basis. Therefore, when designing the facility network, it is essential to study how clients choose the facilities to patronize. The authors propose two alternative mixed integer models to study the impact of client choice behavior on the configuration of a preventive care facility network and the resulting level of participation. Problems are solved with a probabilistic search algorithm and a genetic algorithm. The models are used to analyze the network of mammography centers in Montreal.

\section{Comparison of centralized and focused clinics}

P.T. Vanberkel, R.J. Boucherie, E.W. Hans, J.L. Hurink and N. Litvak compare centralized clinics where health care resources are pooled according to function to achieve economies of scale with focused clinics where health care resources are organized around patient groups such as breast cancer patients. Using queueing theory and simulation, the authors derive a number of interesting results under what circumstances each of the two settings, pooled versus focused, is more advantageous.

\section{Analytical models to determine room requirements in outpatient clinics}

P.J.H. Hulshof, P.T. Vanberkel, R.J. Boucherie, E.W. Hans, M. van Houdenhoven and J.-K.C.W. van Ommeren consider the question of how many examination rooms to assign to a doctor working in an ambulatory clinic. The problem arises when patients have some activities that do not require the doctor to be present such as when a nurse performs a preliminary consult. Since all times are stochastic, the authors used 
queueing theory to find the optimal trade-off between doctor utilization, access time, and patient wait time.

\section{Scheduling of surgeries}

The paper of I. Marques, M.E. Captivo and M.V. Pato addresses the popular topic of elective surgery scheduling. In particular, they study the problem of simultaneous advance and allocation scheduling, thus assigning elective surgeries to an operating room, day, and period to maximize utilization. The authors develop an integer linear programming approach and improvement heuristics, and test these on a real case of a hospital in Lisbon. The results are benchmarked against the actual performance of the hospital's surgical suite.

\section{The single-day surgery scheduling problem}

W.L. Herring and J.W. Herrmann propose a new approach to surgery scheduling. In practice, many hospitals use a threshold date to release unused operating room time. They know that high priority cases continue to arrive, and allocating surgical time to lower priority patients could create problems on the actual day. To make more equitable waiting list decisions, the authors recommend that operating room managers should gradually release unused OR time over the course of several days leading up to the day of surgery.

\section{Scheduling treatments in rehabilitation hospitals}

K. Schimmelpfeng, S. Helber and S. Kasper consider the problem of scheduling treatments in rehabilitation hospitals. This problem has several unique characteristics such as fixed and deterministic processing times, the assignment of a specific resource to a specific patient for a series of treatments, and scheduling of collective activities such as groups of patients for some joint therapy. The authors propose a large-scale mixed integer linear program (MILP) which, due to its size, is hierarchically decomposed into a three-stage model. By experimental comparison of the MILP and the decomposition approach, it is shown that the latter is capable of solving large real-world problems without sacrificing too much solution quality.

Open Access This article is distributed under the terms of the Creative Commons Attribution License which permits any use, distribution, and reproduction in any medium, provided the original author(s) and the source are credited. 